Network Working Group

Request for Comments: 3434

Category: Standards Track
A. Bierman K. McCloghrie

Cisco Systems, Inc. December 2002

\title{
Remote Monitoring MIB Extensions for High Capacity Alarms
}

\section{Status of this Memo}

This document specifies an Internet standards track protocol for the Internet community, and requests discussion and suggestions for improvements. Please refer to the current edition of the "Internet Official Protocol Standards" (STD 1) for the standardization state and status of this protocol. Distribution of this memo is unlimited.

Copyright Notice

Copyright (C) The Internet Society (2002). All Rights Reserved.

Abstract

This memo defines a portion of the Management Information Base (MIB) for use with network management protocols in the Internet community. In particular, it describes managed objects for extending the alarm thresholding capabilities found in the Remote Monitoring (RMON) MIB (RFC 2819), to provide similar threshold monitoring of objects based on the Counter64 data type.

Table of Contents

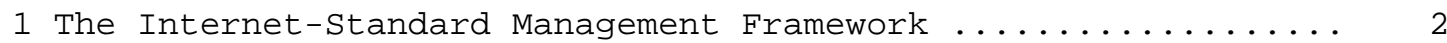

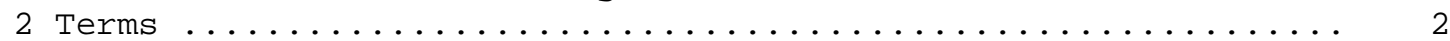

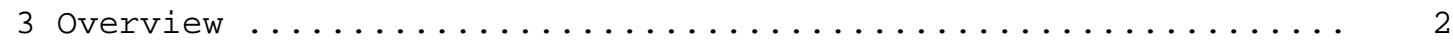

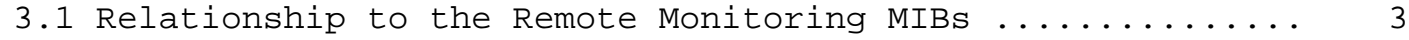

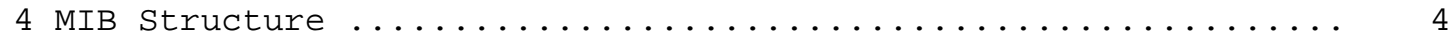

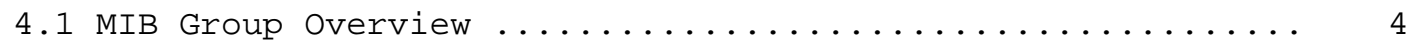

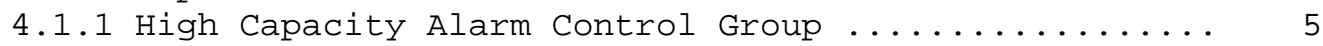

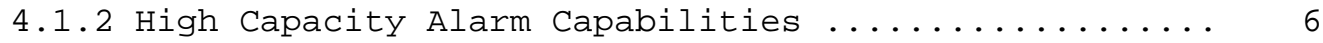

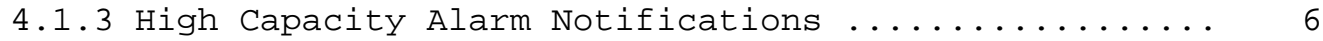

5 Definitions .............................. 6

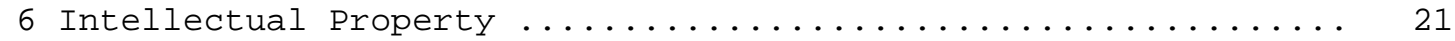

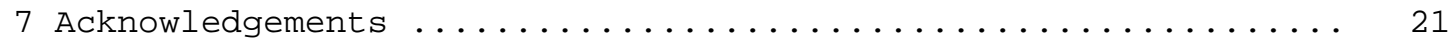

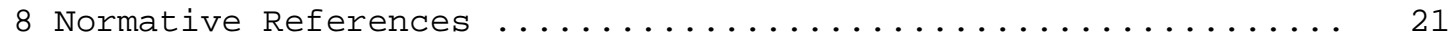

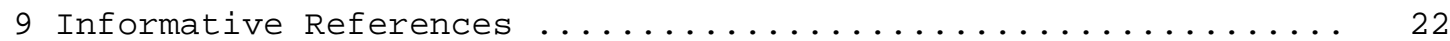




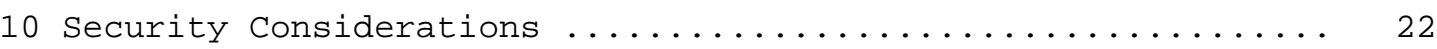

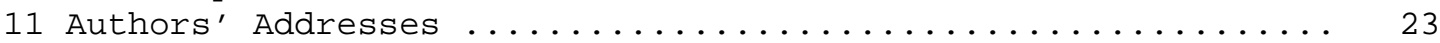

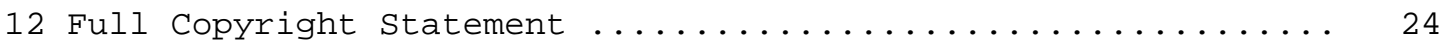

1. The Internet-Standard Management Framework

For a detailed overview of the documents that describe the current Internet-Standard Management Framework, please refer to section 7 of RFC 3410 [RFC3410].

Managed objects are accessed via a virtual information store, termed the Management Information Base or MIB. MIB objects are generally accessed through the Simple Network Management Protocol (SNMP). objects in the MIB are defined using the mechanisms defined in the Structure of Management Information (SMI). This memo specifies a MIB module that is compliant to the SMIV2, which is described in STD 58, RFC 2578 [RFC2578], STD 58, RFC 2579 [RFC2579] and STD 58, RFC 2580 [RFC2580].

2. Terms

The key words "MUST", "MUST NOT", "REQUIRED", "SHALL", "SHALL NOT", "SHOULD", "SHOULD NOT", "RECOMMENDED", "MAY", and "OPTIONAL" in this document are to be interpreted as described in BCP 14, RFC 2119. [RFC2119]

3. Overview

There is a need for a standardized way of providing the same type of alarm thresholding capabilities for Counter64 objects, as already exists for Counter32 objects. The RMON-1 alarmTable objects and RMON-1 notification types are specific to 32-bit objects, and cannot be used to properly monitor Counter64-based objects. Extensions to these existing constructs which explicitly support Counter64-based objects are needed. These extensions are completely independent of the existing RMON-1 alarm mechanisms.

The usage of Counter64 objects is increasing. One of the causes for this increase is the increasing speeds of network interfaces; RFC 2863 [RFC2863] says:

As the speed of network media increase, the minimum time in which a 32 bit counter will wrap decreases. For example, a 10Mbs stream of back-to-back, full-size packets causes ifInoctets to wrap in just over 57 minutes; at $100 \mathrm{Mbs}$, the minimum wrap time is 5.7 minutes, and at $1 \mathrm{Gbs}$, the minimum is 34 seconds. Requiring that interfaces be polled frequently enough not to miss a counter wrap is increasingly problematic. 
and therefore requires:

For interfaces that operate at 20,000,000 (20 million) bits per second or less, 32-bit byte and packet counters MUST be supported. For interfaces that operate faster than 20,000,000 bits/second, and slower than 650,000,000 bits/second, 32-bit packet counters MUST be supported and 64-bit octet counters MUST be supported. For interfaces that operate at 650,000,000 bits/second or faster, 64-bit packet counters AND 64-bit octet counters MUST be supported.

Of the variables on which thresholds are set using RMON-1's alarmTable, two of the most popular are: ifInoctets and ifoutoctets. Thus, the increasing usage of the 64-bit versions: ifHCInoctets and ifHCOutOctets means that there is an increasing requirement to use RMON-1's thresholding capability for ifHCInOctets and ifHCOutOctets.

The RMON-1 Alarm Group is implemented not only by all RMON probes, but also by the SNMP agents in many other types of devices for the purpose of monitoring any of their (non-RMON) integer-valued MIB objects. The fact that it has been so widely implemented indicates its obvious value. Without this extension, that obvious value is becoming incomplete because of its lack of support for 64-bit integers. This extension is the easiest, simplest, and most compatible way for an implementation to overcome that lack of support.

\subsection{Relationship to the Remote Monitoring MIBs}

This MIB is intended to be implemented in Remote Monitoring (RMON) probes, which may also support the RMON-1 MIB [RFC2819]. Such probes may be stand-alone devices, or may be co-located with other networking devices (e.g., ethernet switches and repeaters).

The functionality of the High Capacity Alarm Group is a superset of RMON-1's Alarm Group. Thus, one day in the distant future, it is a possibility that RMON-1's Alarm Group will be deprecated in favor of this MIB's High Capacity Alarm Group. However, that day will not come before this document, or one of its successors, reaches the same standardization state as RMON-1. 
4. MIB structure

Figure 1: HC-ALARM MIB Functional Structure

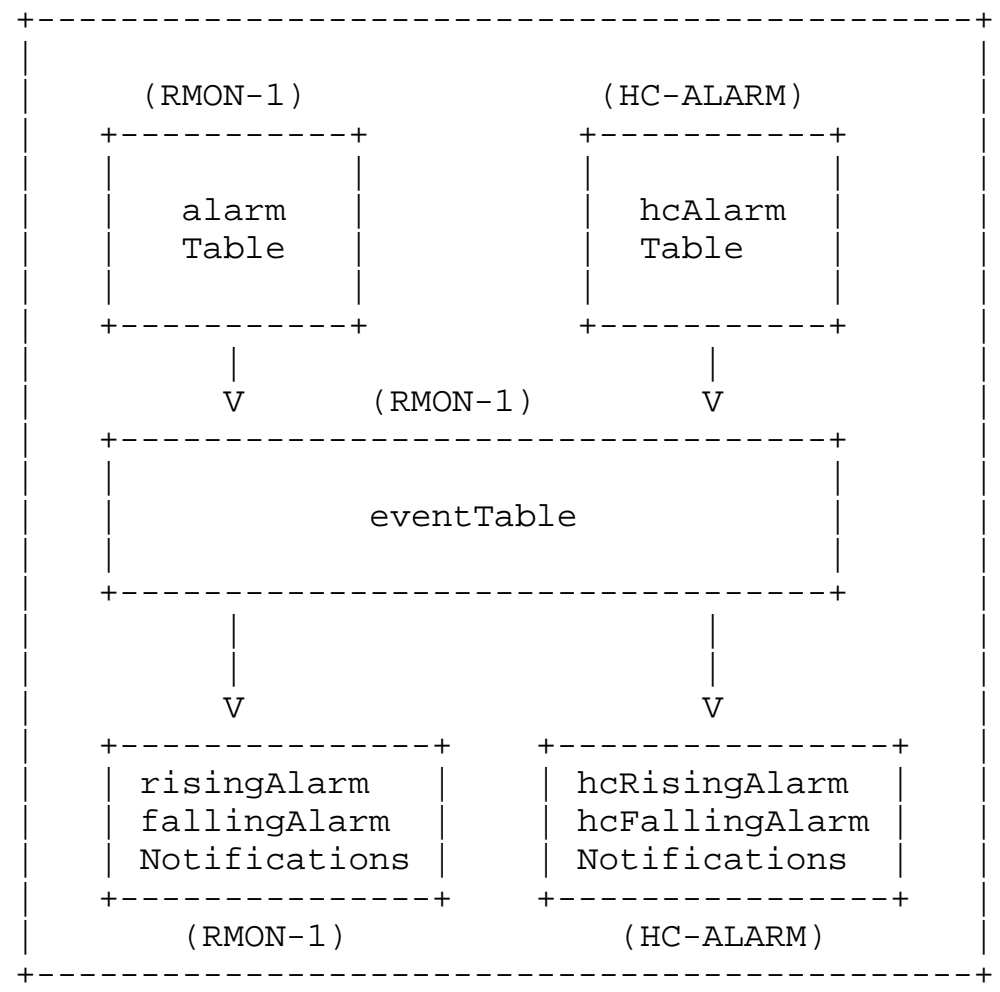

4.1. MIB Group Overview

The HC-ALARM MIB contains three MIB groups:

- hcAlarmControlobjects group Controls the configuration of alarms for high capacity MIB object instances.

- hcAlarmCapabilities group Describes the high capacity alarm capabilities provided by the agent.

- hcAlarmNotifications group Provide new rising and falling threshold notifications for high capacity objects. 


\subsubsection{High Capacity Alarm Control Group}

This group contains one table, which is used by a management station to configure high capacity alarm entries. To configure alarm thresholding for Counter64 or CounterBasedGauge64 objects, a management application must configure the hcAlarmTable in a manner similar to how RMON-1's alarmTable is configured.

Because the language in some of the DESCRIPTION clauses of objects in the alarmTable is specific to the alarmTable itself, their defined semantics do not allow them to be used for this MIB also. Therefore, the following objects are essentially cloned from the alarmTable to the hcAlarmTable:

$\begin{array}{ll}\text { alarmTable } & \text { hcAlarmTable } \\ ------- & -------- \\ \text { alarmIndex } & \text { hcAlarmIndex } \\ \text { alarmInterval } & \text { hcAlarmInterval } \\ \text { alarmVariable } & \text { hcAlarmVariable } \\ \text { alarmSampleType } & \text { hcAlarmSampleType } \\ \text { alarmStartupAlarm } & \text { hcAlarmStartupAlarm } \\ \text { alarmRisingEventIndex } & \text { hcAlarmRisingEventIndex } \\ \text { alarmFallingEventIndex } & \text { hcAlarmFallingEventIndex } \\ \text { alarmOwner } & \text { hcAlarmOwner } \\ \text { alarmStatus } & \text { hcAlarmStatus }\end{array}$

In addition, the following hcAlarmTable objects are used as high capacity values instead of the corresponding 32-bit version in the alarmTable.

$\begin{array}{ll}\text { alarmiable } & \text { hcAlarmTable } \\ ------- & -------- \\ \text { alarmValue } & \text { hcAlarmAbsValue } \\ \text { alarmRisingThreshold } & \text { hcAlarmValueStatus } \\ & \text { hcAlarmRisingThreshAbsValueLo } \\ & \text { hcAlarmRisingThreshAbsValueHi } \\ \text { alarmFallingThreshold } & \text { hcAlarmRisingThresholdValstatus } \\ & \text { hcAlarmFallingThreshAbsValueLo } \\ & \text { hcAlarmFallingThreshAbsValueHi } \\ & \text { hcAlarmFallingThresholdValstatus }\end{array}$

Nevertheless, the hcAlarmTable does have a few differences from the alarmTable:

- Counter64 based objects are thresholded properly

- an entry is not destroyed if the instance identified by the hcAlarmVariable is not available during a polling interval. 
- the RowStatus textual convention is used instead of Entrystatus for the hcAlarmstatus object.

- the non-volatile storage of an HC alarm entry is explicitly controlled with a storageType parameter.

- a counter is provided to indicate the number of times the hcAlarmvariable object value could not be retrieved by the agent.

\subsubsection{High Capacity Alarm Capabilities}

This group contains a single scalar object, called hcAlarmcapabilities. It describes the basic high capacity alarm features supported by the agent.

\subsubsection{High Capacity Alarm Notifications}

This group contains two notifications, hcRisingAlarm and hcFallingAlarm. These are generated for high capacity alarms in the same manner and used to convey essentially the same information as RMON-1's risingAlarm and fallingAlarm notifications do for alarmiable-specified alarms.

5. Definitions

HC-ALARM-MIB DEFINITIONS : := BEGIN

IMPORTS

MODULE-IDENTITY, OBJECT-TYPE, NOTIFICATION-TYPE, Integer32, Counter32, Unsigned32 FROM SNMPV2-SMI MODULE-COMPLIANCE, OBJECT-GROUP, NOTIFICATION-GROUP FROM SNMPV2-CONF

Rowstatus, Variablepointer, StorageType, TEXTUAL-CONVENTION FROM SNMPV2-TC

CounterBasedGauge 64 FROM HCNUM-TC

rmon, OwnerString, rmonEventGroup FROM RMON-MIB;

hCAlarmMIB MODULE-IDENTITY

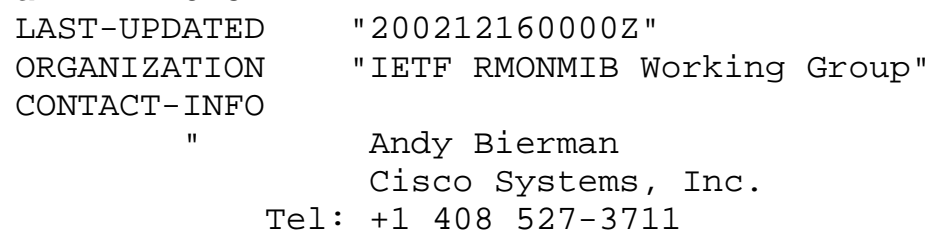




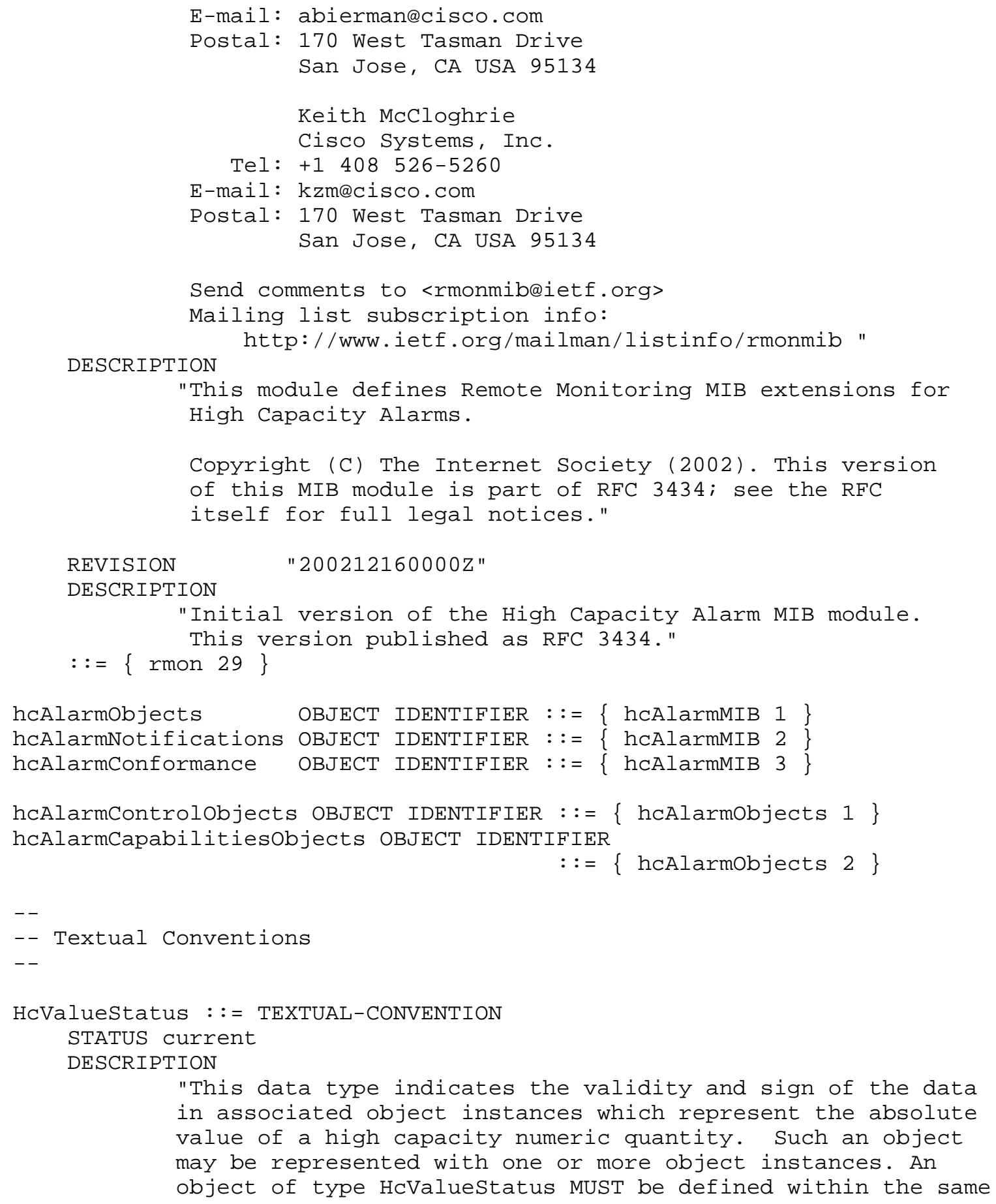




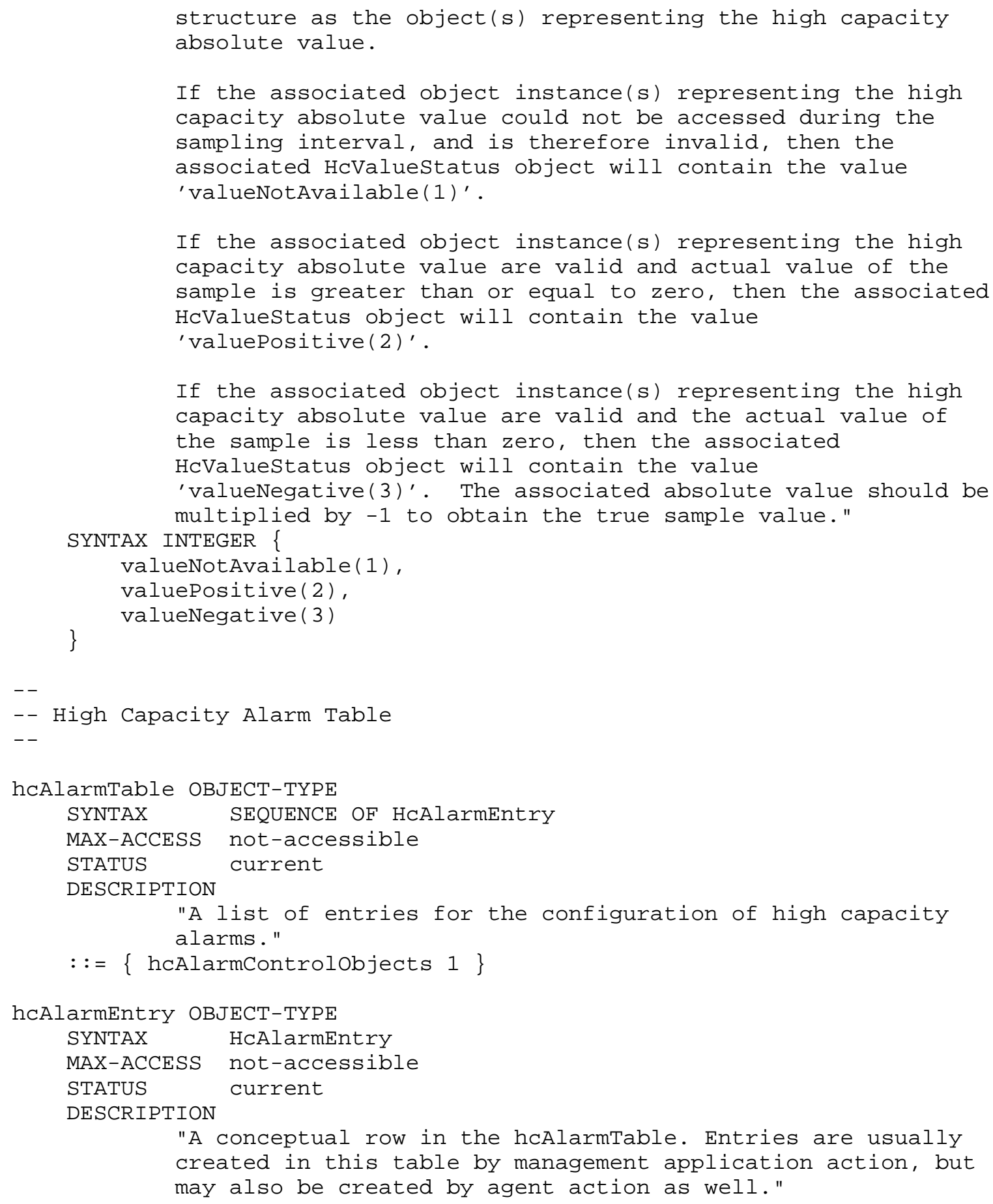


INDEX \{ hcAlarmIndex $\}$ $::=\{$ hcAlarmTable 1$\}$

HCAlarmentry ::= SEQUENCE \{

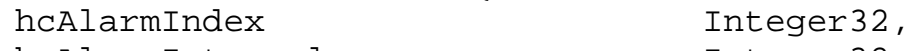


This object may not be modified if the associated

hcAlarmstatus object is equal to active(1)."

$::=\{$ hcAlarmentry 2$\}$

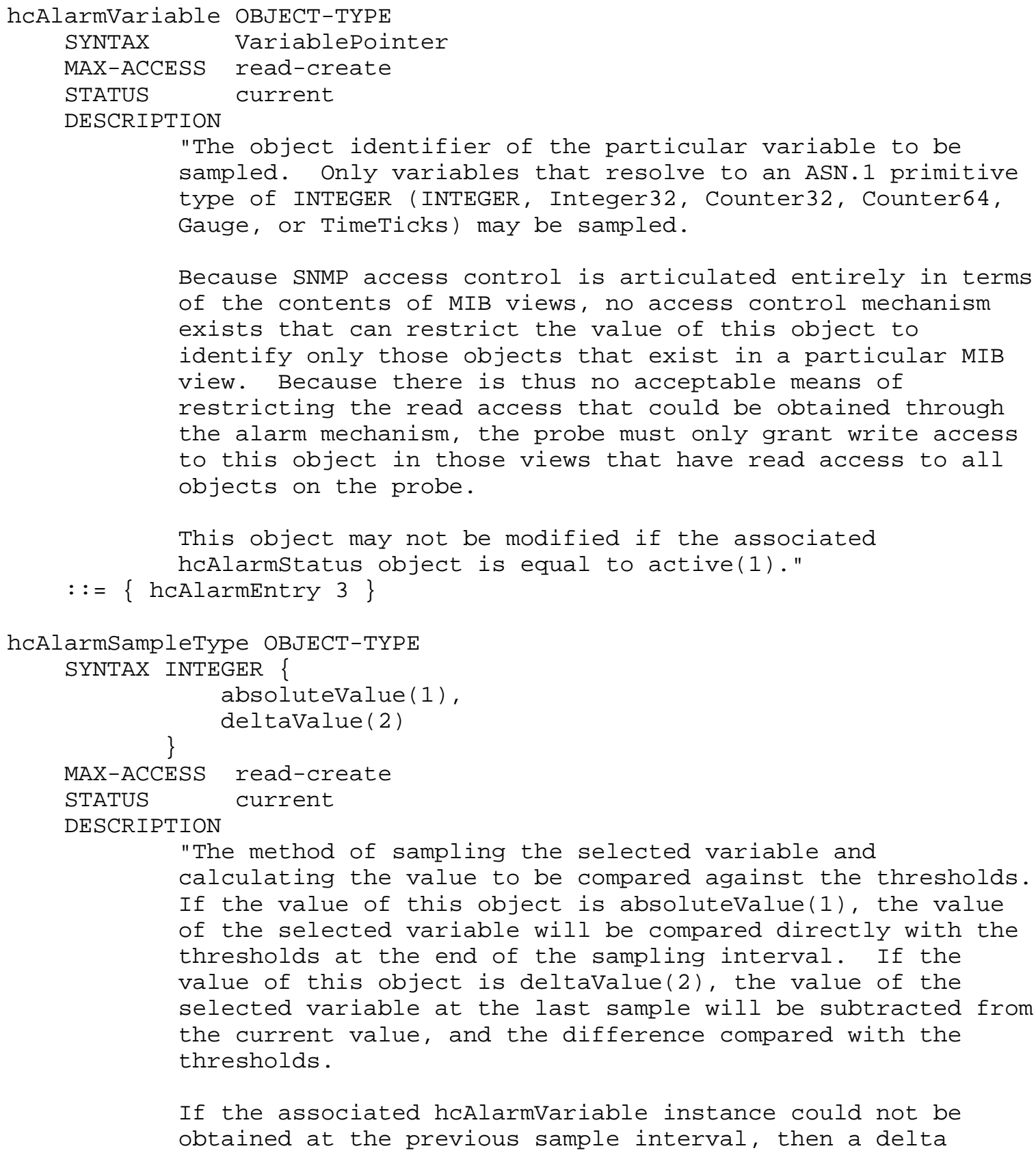




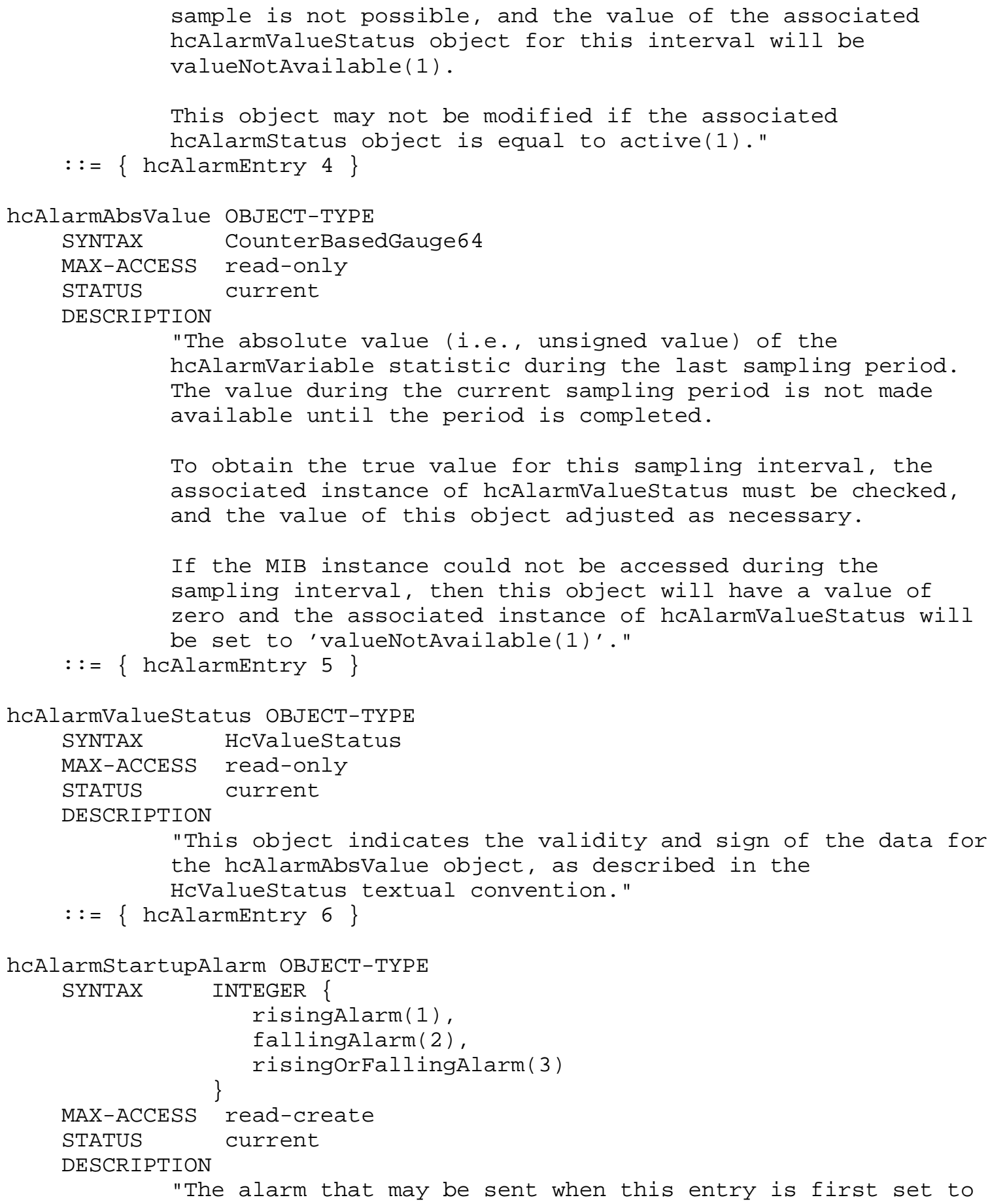


active. If the first sample after this entry becomes active is greater than or equal to the rising threshold and this object is equal to risingAlarm(1) or

risingOrFallingAlarm(3), then a single rising alarm will be generated. If the first sample after this entry becomes valid is less than or equal to the falling threshold and this object is equal to fallingAlarm(2) or risingorfallingAlarm(3), then a single falling alarm will be generated.

This object may not be modified if the associated hcAlarmstatus object is equal to active(1)." $::=\{$ hcAlarmEntry 7$\}$

hcAlarmRisingThreshAbsValueLo OBJECT-TYPE SYNTAX Unsigned32 MAX-ACCESS read-create STATUS current DESCRIPTION

"The lower 32 bits of the absolute value for threshold for the sampled statistic. The actual threshold value is determined by the associated instances of the hcAlarmRisingThreshAbsValueHi and hcAlarmRisingThresholdValstatus objects, as follows:

ABS (threshold) = hcAlarmRisingThreshAbsValueLo + (hcAlarmRisingThreshAbsValueHi * 2^^32)

The absolute value of the threshold is adjusted as required, as described in the HcValuestatus textual convention. These three object instances are conceptually combined to represent the rising threshold for this entry.

When the current sampled value is greater than or equal to this threshold, and the value at the last sampling interval was less than this threshold, a single event will be generated. A single event will also be generated if the first sample after this entry becomes valid is greater than or equal to this threshold and the associated hcAlarmStartupAlarm is equal to risingAlarm(1) or risingorFallingAlarm (3) .

After a rising event is generated, another such event will not be generated until the sampled value falls below this threshold and reaches the threshold identified by the hcAlarmFallingThreshAbsValueLo, hcAlarmFallingThreshAbsValueHi, and hcAlarmFallingThresholdValstatus objects. 
This object may not be modified if the associated hcAlarmstatus object is equal to active(1)."

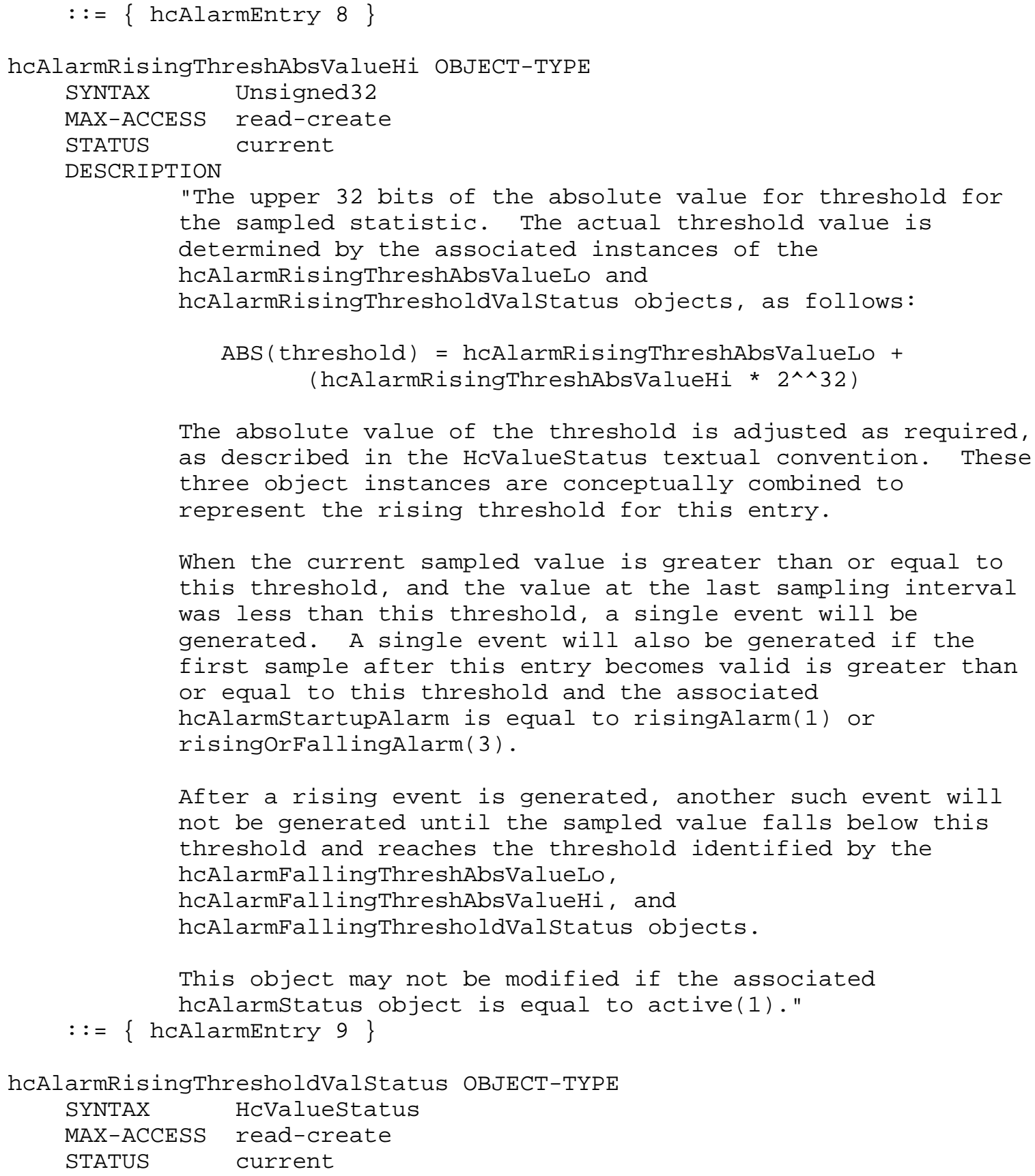




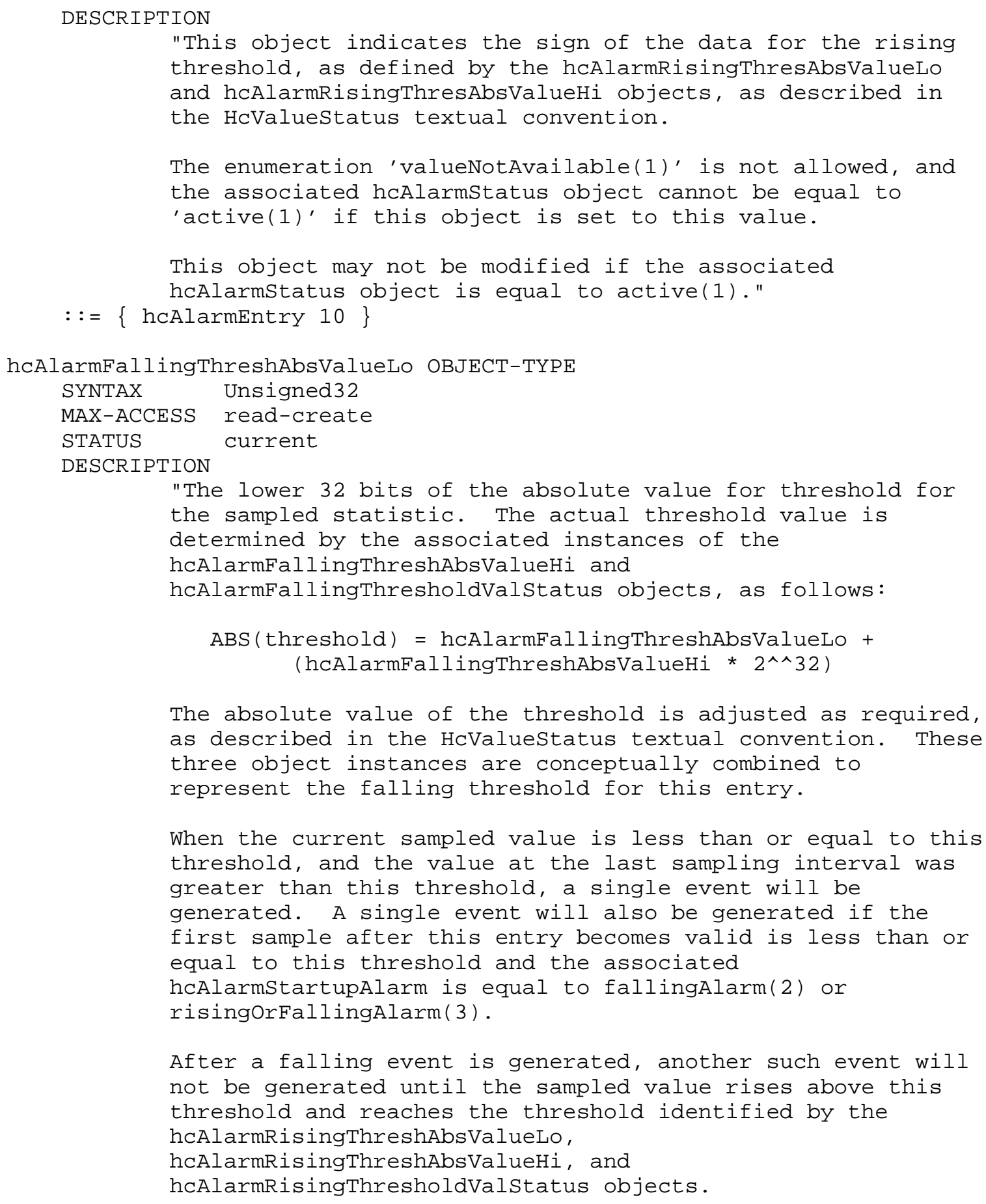


This object may not be modified if the associated

hcAlarmstatus object is equal to active(1)."

$::=\{$ hcAlarmentry 11$\}$

hcAlarmFallingThreshAbsValueHi OBJECT-TYPE

SYNTAX Unsigned32

MAX-ACCESS read-create

STATUS current

DESCRIPTION

"The upper 32 bits of the absolute value for threshold for

the sampled statistic. The actual threshold value is

determined by the associated instances of the

hcAlarmFallingThreshAbsValueLo and

hcAlarmfallingThresholdValstatus objects, as follows:

ABS (threshold) = hcAlarmFallingThreshAbsValueLo +

(hcAlarmFallingThreshAbsValueHi * 2^^32)

The absolute value of the threshold is adjusted as required, as described in the HcValuestatus textual convention. These three object instances are conceptually combined to

represent the falling threshold for this entry.

When the current sampled value is less than or equal to this threshold, and the value at the last sampling interval was greater than this threshold, a single event will be generated. A single event will also be generated if the first sample after this entry becomes valid is less than or equal to this threshold and the associated hcAlarmStartupAlarm is equal to fallingAlarm(2) or risingorfallingAlarm (3).

After a falling event is generated, another such event will not be generated until the sampled value rises above this threshold and reaches the threshold identified by the hcAlarmRisingThreshAbsValueLo, hcAlarmRisingThreshAbsValueHi, and hcAlarmRisingThresholdValstatus objects.

This object may not be modified if the associated hcAlarmstatus object is equal to active(1)." $::=\{$ hcAlarmentry 12$\}$

hCAlarmFallingThresholdValstatus OBJECT-TYPE SYNTAX HCValuestatus

MAX-ACCESS read-create

STATUS current

DESCRIPTION 


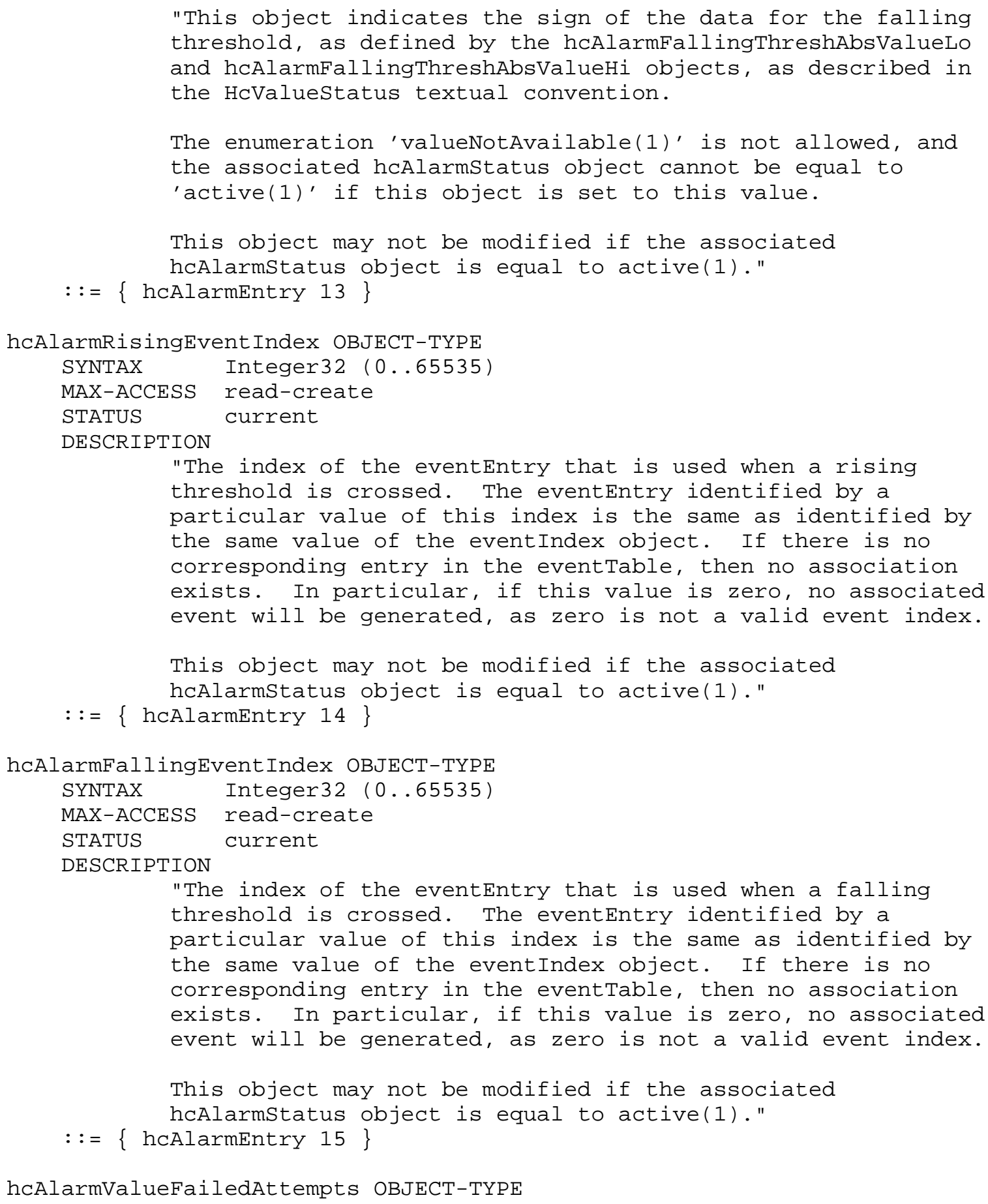




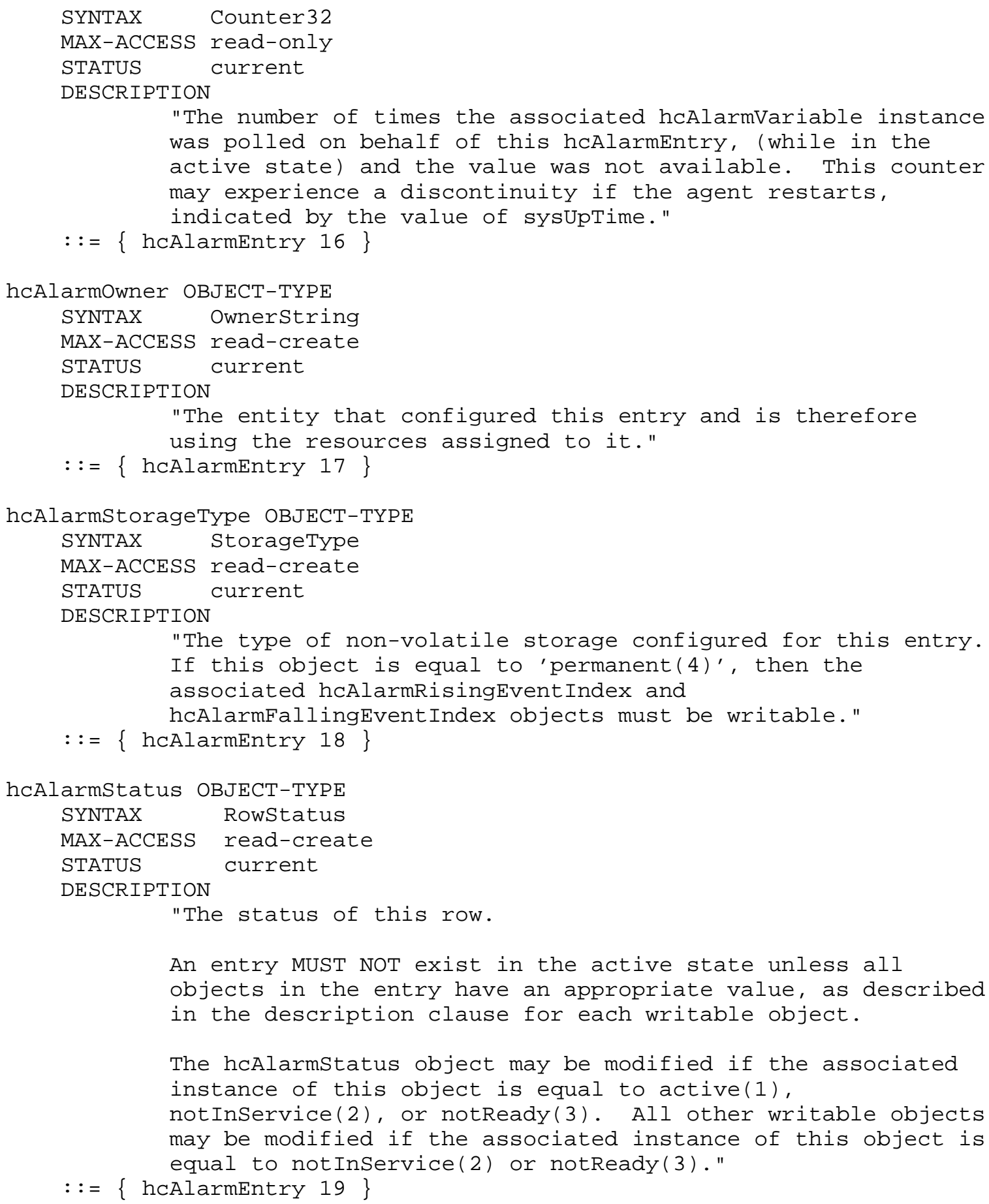




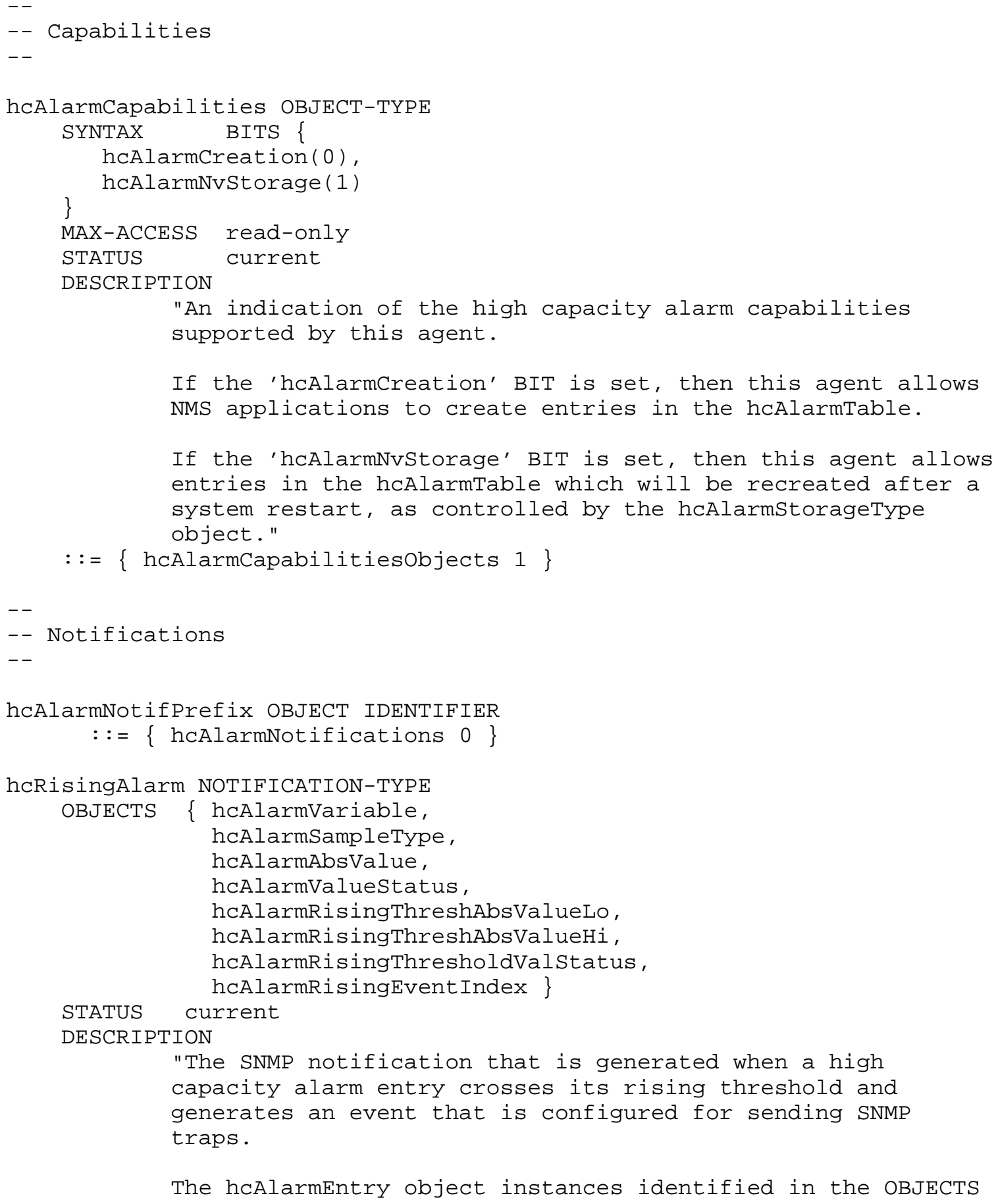




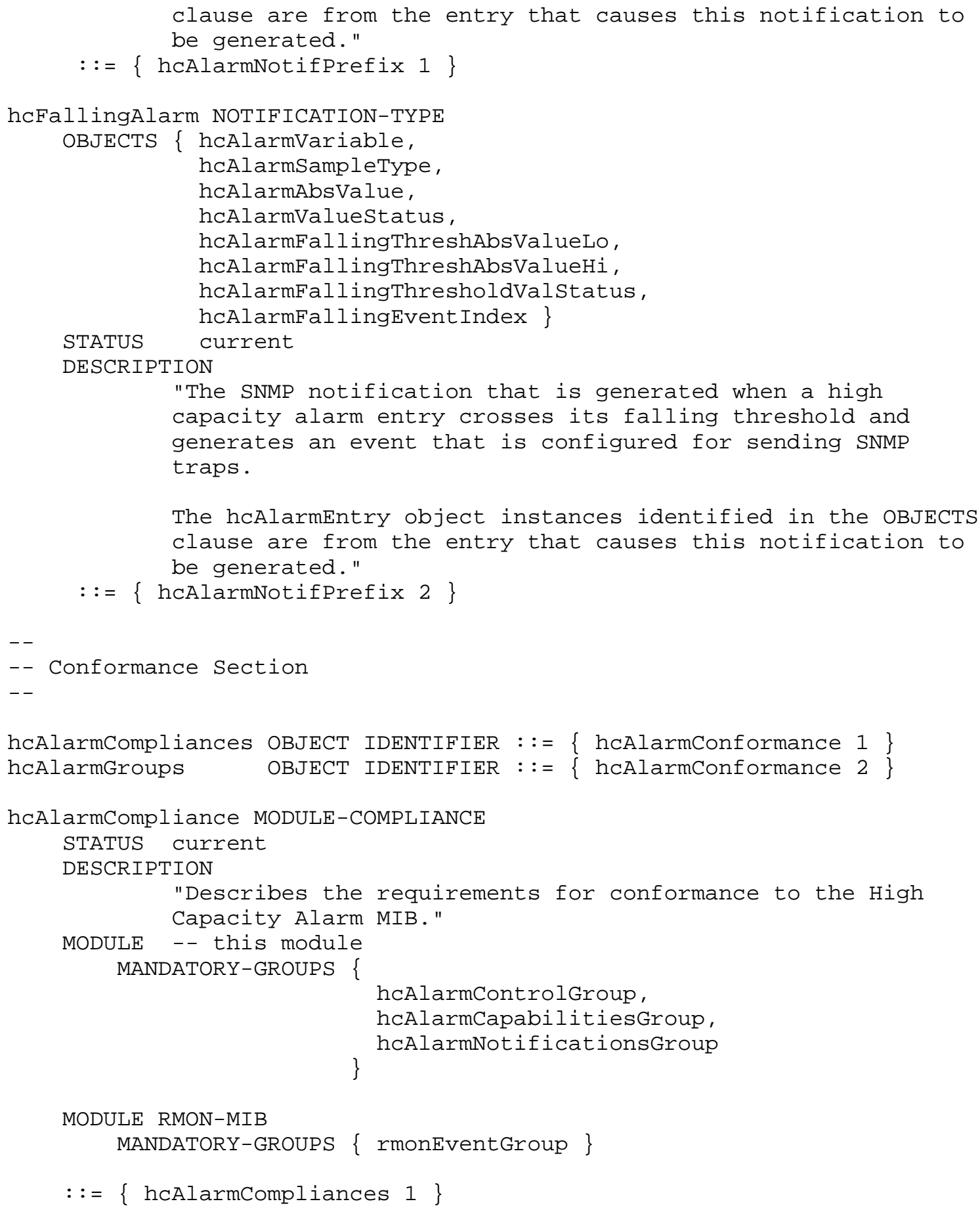


-- Object Groups

hCAlarmControlGroup OBJECT-GROUP OBJECTS \{

hcAlarminterval, hcAlarmVariable, hcAlarmSampleType, hcAl armAbsValue, hcAlarmValuestatus, hcAlarmStartupAlarm, hcAlarmRisingThreshAbsValueLo, hcAlarmRisingThreshAbsValueHi, hcAlarmRisingThresholdValstatus, hcAlarmFallingThreshAbsValueLo, hcAlarmFallingThreshAbsValueHi, hcAlarmFallingThresholdValstatus, hcAlarmRisingEvent Index, hcAlarmFallingEvent Index, hcAlarmValueFailedAt empts, hcAlarmowner, hcAlarmStorageType,

hCAlarmNotificationsGroup NOTIFICATION-GROUP NOTIFICATIONS \{ hcRisingAlarm, hcFallingAlarm

\} STATUS current DESCRIPTION

"A collection of notifications to deliver information related to a high capacity rising or falling threshold event 


$$
::=\left\{\begin{array}{c}
\text { to a management application." } \\
:=\{\text { hcAlarmGroups } 3\}
\end{array}\right.
$$

END

6. Intellectual Property

The IETF takes no position regarding the validity or scope of any intellectual property or other rights that might be claimed to pertain to the implementation or use of the technology described in this document or the extent to which any license under such rights might or might not be available; neither does it represent that it has made any effort to identify any such rights. Information on the IETF's procedures with respect to rights in standards-track and standards-related documentation can be found in BCP-11. Copies of claims of rights made available for publication and any assurances of licenses to be made available, or the result of an attempt made to obtain a general license or permission for the use of such proprietary rights by implementors or users of this specification can be obtained from the IETF Secretariat.

The IETF invites any interested party to bring to its attention any copyrights, patents or patent applications, or other proprietary rights which may cover technology that may be required to practice this standard. Please address the information to the IETF Executive Director.

7. Acknowledgements

This memo is a product of the RMONMIB working group, and is based on existing alarmTable objects in the RMON-1 MIB module [RFC2819]. In order to maintain the RMON 'look-and-feel' and semantic consistency, some of Steve Waldbusser's text from [RFC2819] has been adapted for use in this MIB.

8. Normative References

[RFC2026] Bradner, S., "The Internet Standards Process -- Revision 3", BCP 9, RFC 2026, October 1996.

[RFC2119] Bradner, S., "Key words for use in RFCs to Indicate Requirement Levels", BCP 14, RFC 2119, March 1997. 
[RFC2578] McCloghrie, K., Perkins, D., Schoenwaelder, J., Case, J., Rose, M. and S. Waldbusser, "Structure of Management Information Version 2 (SMIV2)", STD 58, RFC 2578, April 1999.

[RFC2579] McCloghrie, K., Perkins, D., Schoenwaelder, J., Case, J., Rose, M. and S. Waldbusser, "Textual Conventions for SMIv2", STD 58, RFC 2579, April 1999.

[RFC2580] McCloghrie, K., Perkins, D., Schoenwaelder, J., Case, J., Rose, M. and S. Waldbusser, "Conformance Statements for SMIV2", RFC 2580, STD 58, April 1999.

[RFC2819] Waldbusser, S., "Remote Network Monitoring Management Information Base", STD 59, RFC 2819, May 2000.

[RFC3414] Blumenthal, U. and B. Wijnen, "User-based Security Model (USM) for version 3 of the Simple Network Management Protocol (SNMPv3)", STD 62, RFC 3414, December 2002.

[RFC3415] Wijnen, B., Presuhn, R. and K. McCloghrie, "View-based Access Control Model (VACM) for the Simple Network Management Protocol (SNMP)", STD 62, RFC 3415, December 2002 .

9. Informative References

[RFC3410] Case, J., Mundy, R., Partain, D. and B. Stewart, "Introduction and Applicability Statements for InternetStandard Management Framework", RFC 3410, December 2002.

[RFC2863] McCloghrie, K. and F. Kastenholz, "The Interfaces Group MIB", RFC 2863, June, 2000.

10. Security Considerations

There are a number of management objects defined in this MIB that have a MAX-ACCESS clause of read-write and/or read-create. Such objects may be considered sensitive or vulnerable in some network environments. The support for SET operations in a non-secure environment without proper protection can have a negative effect on network operations.

There are a number of managed objects in this MIB that may contain sensitive information. These are:

hcAlarmAbsValue

hcAlarmvaluestatus 
These objects are used together, and may expose the values of particular MIB instances, as identified by associated instances of the hcAlarmVariable object.

hcAlarmVariable

This object identifies the object instance that the associated hcAlarmEntry will periodically sample. Because SNMP access control is articulated entirely in terms of the contents of MIB views, no access control mechanism exists that can restrict the value of this object to identify only those objects that exist in a particular MIB view. Thus, because there is no acceptable means of restricting the read access that could be obtained through the alarm mechanism, the probe must only grant write access to this object in those views that have read access to all objects on the probe.

SNMPV1 by itself is not a secure environment. Even if the network itself is secure (for example by using IPSec), there is no control as to who on the secure network is allowed to access and GET/SET (read/change/create/delete) the objects in this MIB.

It is recommended that the implementors consider the security features as provided by the SNMPv3 framework. Specifically, the use of the User-based Security Model STD 62, RFC 3414 [RFC3414] and the View-based Access Control Model STD 62, RFC 3415 [RFC3415] is recommended.

It is then a customer/user responsibility to ensure that the SNMP entity giving access to an instance of this MIB, is properly configured to give access to only the objects, and to those principals (users) that have legitimate rights to indeed GET or SET (change/create/delete) them.

\section{Authors' Addresses}

Andy Bierman

Cisco Systems, Inc.

170 West Tasman Drive

San Jose, CA USA 95134

Phone: +1 408-527-3711

EMail: abiermandcisco.com

Keith McCloghrie

Cisco Systems, Inc.

170 West Tasman Drive

San Jose, CA USA 95134

Phone: +1 408-526-5260

EMail: kzmecisco.com 
12. Full Copyright statement

Copyright (C) The Internet Society (2002). All Rights Reserved.

This document and translations of it may be copied and furnished to others, and derivative works that comment on or otherwise explain it or assist in its implementation may be prepared, copied, published and distributed, in whole or in part, without restriction of any kind, provided that the above copyright notice and this paragraph are included on all such copies and derivative works. However, this document itself may not be modified in any way, such as by removing the copyright notice or references to the Internet society or other Internet organizations, except as needed for the purpose of developing Internet standards in which case the procedures for copyrights defined in the Internet Standards process must be followed, or as required to translate it into languages other than English.

The limited permissions granted above are perpetual and will not be revoked by the Internet society or its successors or assigns.

This document and the information contained herein is provided on an "AS IS" basis and THE INTERNET SOCIETY AND THE INTERNET ENGINEERING TASK FORCE DISCLAIMS ALL WARRANTIES, EXPRESS OR IMPLIED, INCLUDING BUT NOT LIMITED TO ANY WARRANTY THAT THE USE OF THE INFORMATION HEREIN WILL NOT INFRINGE ANY RIGHTS OR ANY IMPLIED WARRANTIES OF MERCHANTABILITY OR FITNESS FOR A PARTICULAR PURPOSE.

Acknowledgement

Funding for the RFC Editor function is currently provided by the Internet society. 